\title{
Relativistic interacting integrable elliptic tops
}

\author{
Andrei Zotov \\ Steklov Mathematical Institute of Russian Academy of Sciences, \\ Gubkina str. 8, Moscow, 119991, Russia \\ E-mail: zotov@mi-ras.ru
}

\begin{abstract}
We propose relativistic generalization of integrable systems describing $M$ interacting elliptic $\operatorname{gl}(N)$ tops of the Euler-Arnold type. The obtained models are elliptic integrable systems, which reproduce the spin elliptic $\operatorname{GL}(M)$ Ruijsenaars-Schneider model for $N=1$ case, while in the $M=1$ case they turn into relativistic integrable $\mathrm{GL}(N)$ elliptic tops. The Lax pairs with spectral parameter on elliptic curve are constructed.
\end{abstract}

\section{Introduction}

In [8] Krichever and Zabrodin suggested the following ansatz for the Lax pair with spectral parameter of the spin elliptic GL $(M)$ Ruijsenaars-Schneider model:

$$
\begin{gathered}
L_{i j}(z)=S_{i j} \phi\left(z, q_{i j}+\eta\right), \quad i, j=1, \ldots, M \\
q_{i j}=q_{i}-q_{j}, \quad \operatorname{Res}_{z=0} L(z)=S \in \operatorname{Mat}(M, \mathbb{C}), \\
M_{i j}(z)=-\delta_{i j}\left(E_{1}(z)+E_{1}(\eta)\right) S_{i i}-\left(1-\delta_{i j}\right) S_{i j} \phi\left(z, q_{i j}\right) .
\end{gathered}
$$

The definitions of the Kronecker function $\phi$ and elliptic functions $E_{1}, E_{2}$ are given in the Appendix. Under conditions

$$
S_{i i}=\dot{q}_{i}, \quad i=1, \ldots, M
$$

the Lax equation

$$
\dot{L}(z)=[L(z), M(z)]
$$

provides equations of motion for the diagonal part of matrix $S$

$$
\dot{S}_{i i}=\ddot{q}_{i}=-\sum_{k: k \neq i}^{M} S_{i k} S_{k i}\left(E_{1}\left(q_{i k}+\eta\right)+E_{1}\left(q_{i k}-\eta\right)-2 E_{1}\left(q_{i k}\right)\right)
$$

and for its non-diagonal part (1.4) yields

$$
\dot{S}_{i j}=\sum_{k: k \neq j}^{M} S_{i k} S_{k j}\left(E_{1}\left(q_{k j}+\eta\right)-E_{1}\left(q_{k j}\right)\right)-\sum_{k: k \neq i}^{M} S_{i k} S_{k j}\left(E_{1}\left(q_{i k}+\eta\right)-E_{1}\left(q_{i k}\right)\right),
$$


or, equivalently,

$$
\begin{gathered}
\dot{S}_{i j}=S_{i j}\left(S_{i i}-S_{j j}\right)\left(E_{1}\left(q_{i j}+\eta\right)-E_{1}\left(q_{i j}\right)\right)+ \\
+\sum_{k: k \neq i, j}^{M} S_{i k} S_{k j}\left(E_{1}\left(q_{k j}+\eta\right)-E_{1}\left(q_{k j}\right)-E_{1}\left(q_{i k}+\eta\right)+E_{1}\left(q_{i k}\right)\right) .
\end{gathered}
$$

These equations can be viewed as relativistic deformation [16] (the deformation parameter is $\eta \in \mathbb{C}$ ) of the spin elliptic Calogero-Moser model [3]:

$$
\begin{gathered}
\ddot{q}_{i}=\sum_{k: k \neq i}^{M} S_{i k} S_{k i} E_{2}^{\prime}\left(q_{i k}\right), \\
\dot{S}_{i i}=0, \quad \dot{S}_{i j}=\sum_{k: k \neq i, j}^{M} S_{i k} S_{k j}\left(E_{2}\left(q_{i k}\right)-E_{2}\left(q_{k j}\right)\right) .
\end{gathered}
$$

The system (1.8) admits anisotropic $g l(N M)$ generalization, where the spin variables $S_{i j}$ are replaced by matrix-valued variables $\mathcal{S}^{i j} \in \operatorname{Mat}(N, \mathbb{C})$. Equations of motion are of the form:

$$
\begin{gathered}
\dot{\mathcal{S}}^{i i}=\left[\mathcal{S}^{i i}, J\left(\mathcal{S}^{i i}\right)\right]+\sum_{k: k \neq i}^{M}\left(\mathcal{S}^{i k} J^{q_{k i}}\left(S^{k i}\right)-J^{q_{i k}}\left(\mathcal{S}^{i k}\right) S^{k i}\right) \\
\dot{\mathcal{S}}^{i j}=\mathcal{S}^{i j} J\left(\mathcal{S}^{j j}\right)-J\left(\mathcal{S}^{i i}\right) \mathcal{S}^{i j}+\sum_{k: k \neq j}^{M} \mathcal{S}^{i k} J^{q_{k j}}\left(\mathcal{S}^{k j}\right)-\sum_{k: k \neq i}^{M} J^{q_{i k}}\left(\mathcal{S}^{i k}\right) \mathcal{S}^{k j} \\
\ddot{q}_{i}=-\frac{1}{N} \sum_{k: k \neq i}^{M} \partial_{q_{i}} \operatorname{tr}\left(J^{q_{i k}}\left(\mathcal{S}^{i k}\right) \mathcal{S}^{k i}\right)
\end{gathered}
$$

The anisotropy means the presence (in the equations of motion) of the linear operators $J, J^{q_{i j}}$ acting in the matrix space $\operatorname{Mat}(N, \mathbb{C})$. In the case $N=1$ equations (1.9)-(1.11) reproduce (1.8), while in the case $M=1$ equation (1.9) is simplified to the one of the Euler-Arnold type for the elliptic integrable top [9]:

$$
\dot{S}=[S, J(S)], \quad S \in \operatorname{Mat}(N, \mathbb{C}) .
$$

The systems of type (1.9)-(1.11) appeared in papers [13] in studies of the matrix models, and later they were described as examples of the Hitchin systems on $\operatorname{SL}(N M, \mathbb{C}$ )-bundles (over elliptic curve) with non-trivial characteristic classes [19, 12]. Equations (1.9)-(1.11) for a more general class of the operators $J$ were obtained in [7].

When the matrix of spin variables $\mathcal{S}=\sum_{i j} E_{i j} \otimes \mathcal{S}^{i j} \in \operatorname{Mat}(N M, \mathbb{C})$ is of rank 1, the r.h.s. of equations (1.9) and (1.11) are represented in terms of diagonal blocks of matrix $\mathcal{S}$ only (i.e. in terms of matrices $\mathcal{S}^{i i}$ ):

$$
\dot{\mathcal{S}}^{i i}=\left[\mathcal{S}^{i i}, J\left(\mathcal{S}^{i i}\right)\right]+\sum_{k: k \neq i}^{M}\left[\mathcal{S}^{i i}, \breve{J}^{q i k}\left(\mathcal{S}^{k k}\right)\right], \quad \ddot{q}_{i}=-\frac{1}{N} \sum_{k: k \neq i}^{M} \partial_{q_{i}} \operatorname{tr}\left(\mathcal{S}^{i i} \breve{J}^{q_{i k}}\left(\mathcal{S}^{k k}\right)\right) .
$$

This allows to interpret the equations as dynamics of $M$ interacting tops with positions $q_{i}$. Being written in such a form the model resembles the initial formulation of the (quantum) spin Calogero-Moser model [6]. 
Purpose of the paper is to construct relativistic deformation of the models (1.9)-(1.11) and (1.13). We will show that such generalization exists and has the form:

$$
\begin{gathered}
\dot{\mathcal{S}}^{i i}=\left[\mathcal{S}^{i i}, J^{\eta}\left(\mathcal{S}^{i i}\right)\right]+\sum_{k: k \neq i}^{M}\left(\mathcal{S}^{i k} J^{\eta, q_{k i}}\left(S^{k i}\right)-J^{\eta, q_{i k}}\left(\mathcal{S}^{i k}\right) S^{k i}\right), \\
\dot{\mathcal{S}}^{i j}=\mathcal{S}^{i j} J^{\eta}\left(\mathcal{S}^{j j}\right)-J^{\eta}\left(\mathcal{S}^{i i}\right) \mathcal{S}^{i j}+\sum_{k: k \neq j}^{M} \mathcal{S}^{i k} J^{\eta, q_{k j}}\left(\mathcal{S}^{k j}\right)-\sum_{k: k \neq i}^{M} J^{\eta, q_{i k}}\left(\mathcal{S}^{i k}\right) \mathcal{S}^{k j} \\
\ddot{q}_{i}=\frac{1}{N} \operatorname{tr}\left(\dot{\mathcal{S}}^{i i}\right)=\frac{1}{N} \sum_{k: k \neq i}^{M} \operatorname{tr}\left(\mathcal{S}^{i k} J^{\eta, q_{k i}}\left(\mathcal{S}^{k i}\right)-J^{\eta, q_{i k}}\left(\mathcal{S}^{i k}\right) \mathcal{S}^{k i}\right) .
\end{gathered}
$$

The last equation comes from the trace of both sides of equation (1.14) together with conditions

$$
\dot{q}_{i}=\frac{1}{N} \operatorname{tr}\left(\mathcal{S}^{i i}\right), \quad i=1, \ldots, M
$$

In particular case when the matrix of spin variables is of rank 1 we get the system of $M$ interacting tops associated with $G L(N, \mathbb{C})$ group:

$$
\begin{gathered}
\dot{\mathcal{S}}^{i i}=\left[\mathcal{S}^{i i}, J^{\eta}\left(\mathcal{S}^{i i}\right)\right]+\sum_{k: k \neq i}^{M}\left(\mathcal{S}^{i i} \widetilde{J}^{\eta, q_{k i}}\left(S^{k k}\right)-\breve{J}^{\eta, q_{i k}}\left(\mathcal{S}^{k k}\right) S^{i i}\right), \\
\ddot{q}_{i}=\frac{1}{N} \sum_{k: k \neq i}^{M} \operatorname{tr}\left(\mathcal{S}^{i i} \widetilde{J}^{\eta, q_{k i}}\left(S^{k k}\right)-\breve{J}^{\eta, q_{i k}}\left(\mathcal{S}^{k k}\right) S^{i i}\right) .
\end{gathered}
$$

The obtained models can be viewed as anisotropic matrix generalization of the spin elliptic GL $(M)$ Ruijsenaars-Schneider model, which is reproduced in the $N=1$ case. When $M=1$ we get the relativistic deformation of the elliptic top (1.12), know previously from [10].

The paper is organized as follows. In Sections 2 and 3 we give detailed descriptions of the spin elliptic Ruijsenaars-Schneider model and the relativistic elliptic top respectively. In Section 4 the model (1.14)-(1.16) is described, and $\operatorname{Mat}(N M, \mathbb{C})$-valued Lax representation with spectral parameter on elliptic curve is given. In Section 5 we study the case $\operatorname{rk}(\mathcal{S})=1$ and

obtain equations (1.18)-(1.19). Explicit form of the linear operators $\tilde{J}^{\eta, q_{i j}}$ and $\breve{J}^{\eta, q_{i j}}$ entering (1.18) is derived in the end of Section 5 through the usage of the finite-dimensional Fourier transformation of elliptic functions. The non-relativistic limit is described as well. It is shown that the limit reproduces the previously obtained results [7] in the elliptic case.

\section{Spin Ruijsenaars-Schneider model}

In this Section we derive equations of motion. It will help to simplify the proof of the more complicated statement related to interacting tops. More precisely, we prove the following

Proposition 2.1 Equations of motion

$$
\dot{S}_{i i}=-\sum_{k: k \neq i}^{M} S_{i k} S_{k i}\left(E_{1}\left(q_{i k}+\eta\right)+E_{1}\left(q_{i k}-\eta\right)-2 E_{1}\left(q_{i k}\right)\right)
$$


and (1.6) are equivalent to the Lax equations with additional term:

$$
\dot{L}(z)=[L(z), M(z)]+\sum_{i, j=1}^{M} E_{i j}\left(\mu_{i}-\mu_{j}\right) S_{i j} f\left(z, q_{i j}+\eta\right)
$$

for the pair of matrices (1.1)-(1.2) and the set of variables

$$
\mu_{i}=\dot{q}_{i}-S_{i i}, \quad i=1, \ldots, M .
$$

On-shell the constraints $\mu_{i}=0$ the matrices (1.1)-(1.2) satisfy the Lax equation (1.4) and provide equations of motion (1.5)-(1.6).

Proof. The additional term is absent in the diagonal part of (2.2). Consider the $i$-th diagonal element. In the 1.h.s. of (2.2) in $i i$-th element we have $\dot{S}_{i i} \phi(z, \eta)$, while in the r.h.s. the following expression appears:

$$
\sum_{k: k \neq i} L_{i k} M_{k i}-M_{i k} L_{k i}=\sum_{k: k \neq i} S_{i k} S_{k i}\left(\phi\left(z, q_{i k}\right) \phi\left(z, q_{k i}+\eta\right)-\phi\left(z, q_{i k}+\eta\right) \phi\left(z, q_{k i}\right)\right) .
$$

Equation (2.1) comes from the usage of the relation (A.7). In the off-diagonal part of the equation (2.2) for $i j$-th matrix element (with $i \neq j$ ) in the l.h.s. we have

$$
\dot{S}_{i j} \phi\left(z, q_{i j}+\eta\right)+S_{i j}\left(\dot{q}_{i}-\dot{q}_{j}\right) f\left(z, q_{i j}+\eta\right) .
$$

And in the r.h.s. we get

$$
\begin{gathered}
\left(M_{j j}-M_{i i}\right) L_{i j}+\left(L_{i i}-L_{j j}\right) M_{i j}+ \\
+\sum_{k \neq i, j}\left(L_{i k} M_{k j}-M_{i k} L_{k j}\right)+\left(\mu_{i}-\mu_{j}\right) S_{i j} f\left(z, q_{i j}+\eta\right)= \\
=\left(S_{i i}-S_{j j}\right) S_{i j}\left(E_{1}(z)+E_{1}(\eta)\right) \phi\left(z, q_{i j}+\eta\right)-\left(S_{i i}-S_{j j}\right) S_{i j} \phi\left(z, q_{i j}\right) \phi(z, \eta)- \\
-\sum_{k \neq i, j} S_{i k} S_{k j}\left(\phi\left(z, q_{i k}+\eta\right) \phi\left(z, q_{k j}\right)-\phi\left(z, q_{i k}\right) \phi\left(z, q_{k j}+\eta\right)\right)+\left(\mu_{i}-\mu_{j}\right) S_{i j} f\left(z, q_{i j}+\eta\right) .
\end{gathered}
$$

Transpose the second term of (2.5) from the 1.h.s. of equation (2.2) to its r.h.s. Then the terms proportional to $\left(\dot{q}_{i}-\dot{q}_{j}\right) S_{i j}$ are cancelled out. For the terms proportional to $\left(S_{i i}-S_{j j}\right) S_{i j}$, we get a common factor:

$$
\begin{gathered}
\left(E_{1}(z)+E_{1}(\eta)\right) \phi\left(z, q_{i j}+\eta\right)-\phi\left(z, q_{i j}\right) \phi(z, \eta)-f\left(z, q_{i j}+\eta\right) \stackrel{A .7, \underline{A .8}}{=} \\
=\phi\left(z, q_{i j}+\eta\right)\left(E_{1}\left(q_{i j}+\eta\right)-E_{1}\left(q_{i j}\right)\right) .
\end{gathered}
$$

Using also relation (A.7) for the expression in the sum in (2.6), we finally get the off-diagonal part of equations of motion in the form (1.7). It is easily seen that the latter is equivalent to (1.6). This finishes the proof.

\footnotetext{
${ }^{1}$ The function $f$ entering (2.2) is defined in (A.8), $\left\{E_{i j}\right\}$ - is the standard basis in $\operatorname{Mat}(M, \mathbb{C})$.
} 
The non-relativistic limit appears as follows. Let us redefine the time variable

$$
t \rightarrow t / \eta \text {. }
$$

In particular, it means that $\dot{q}_{i} \mapsto \eta \dot{q}_{i}$ and $\ddot{q}_{i} \mapsto \eta^{2} \ddot{q}_{i}$. From the definition (A.3) near $\eta=0$ we have:

$$
E_{1}(q+\eta)=E_{1}(q)-\eta E_{2}(q)-\frac{1}{2} \eta^{2} E_{2}^{\prime}(q)+O\left(\eta^{3}\right) .
$$

In the limit $\eta \rightarrow 0$ the constraints (1.3) turn into the set of conditions

$$
S_{i i}=0, \quad i=1, \ldots, M,
$$

and the equations of motion (1.5)-(1.7) in view of (2.10) take the form

$$
\ddot{q}_{i}=\sum_{k: k \neq i}^{M} S_{i k} S_{k i} E_{2}^{\prime}\left(q_{i k}\right) .
$$

Equations of motion for the diagonal part of spin variables are $\dot{S}_{i i}=0$, and for $i \neq j$ we have

$$
\dot{S}_{i j}=\sum_{k: k \neq i, j}^{M} S_{i k} S_{k j}\left(E_{2}\left(q_{i k}\right)-E_{2}\left(q_{k j}\right)\right) .
$$

In this way we get the equations of motion of the classical spin Calogero-Moser model [3]. Let us remark that the choice of the constraints $\mu_{i}=0$ is not necessary. All derivations are also valid for the constraints $\mu_{i}=\nu=$ const for all $i=1, \ldots, M$. This is a set of the first class constraints in the Calogero-Moser model. They should be supplied with $M$ conditions of gauge fixation with respect to the coadjoint action of the Cartan subgroup of $\operatorname{GL}(M, \mathbb{C})$, i.e. with respect to conjugation by diagonal matrices. Then the total set of $2 M$ conditions forms the second class constraints, and one should perform the Poisson reduction with respect to these constraints. The reduction procedure changes equations of motion due to reducing the number of independent variables and due to the Dirac terms appearing in the reduced Poisson brackets.

From all has been said it follows that the equations (2.11)-(2.12) should be considered as intermediate stage of the Poisson reduction corresponding to simple restriction of the unreduced system (with linear Poisson-Lie brackets) to the imposed constraints $\mu_{i}=\nu$, but the reduction procedure is not performed yet. The equations of motion in the relativistic case (1.5)-(1.7) should be understood in the same manner on the constraints (1.3). It should be mentioned that the Poisson structure (and the classical $r$-matrix structure) for the spin elliptic RuijsenaarsSchneider model is unknown yet. At the same time for the trigonometric and rational models the Poisson structures and the group-theoretical description are known [1, 15, 5, 4, 2, 14].

\section{Relativistic integrable top}

A special basis in the space $\operatorname{Mat}(N, \mathbb{C})$ (the sine-algebra basis) is used for description of elliptic tops. The basis is of the form

$$
T_{\alpha}=T_{\alpha_{1} \alpha_{2}}=\exp \left(\frac{\pi \imath}{N} \alpha_{1} \alpha_{2}\right) Q^{\alpha_{1}} \Lambda^{\alpha_{2}}, \quad \alpha=\left(\alpha_{1}, \alpha_{2}\right) \in \mathbb{Z}_{N} \times \mathbb{Z}_{N},
$$


where $Q$ and $\Lambda$ - pair of matrices (for which $Q^{N}=\Lambda^{N}=1_{N \times N}$ ) with elements

$$
Q_{k l}=\delta_{k l} \exp \left(\frac{2 \pi \imath}{N} k\right), \quad \Lambda_{k l}=\delta_{k-l+1=0 \bmod N}, \quad k, l=1, \ldots, N .
$$

It is the finite-dimensional representation of the Heisenberg group:

$$
\Lambda^{a_{2}} Q^{a_{1}}=\exp \left(\frac{2 \pi \imath}{N} a_{1} a_{2}\right) Q^{a_{1}} \Lambda^{a_{2}}, \quad a_{1}, a_{2} \in \mathbb{Z} .
$$

It is easy to see that the product of basis matrices can be written in the form

$$
T_{\alpha} T_{\beta}=\kappa_{\alpha, \beta} T_{\alpha+\beta}, \quad \kappa_{\alpha, \beta}=\exp \left(\frac{\pi \imath}{N}\left(\beta_{1} \alpha_{2}-\beta_{2} \alpha_{1}\right)\right)
$$

where $\alpha+\beta=\left(\alpha_{1}+\beta_{1}, \alpha_{2}+\beta_{2}\right)$. In particular, it follows from the latter that

$$
\operatorname{tr}\left(T_{\alpha} T_{\beta}\right)=N \delta_{\alpha,-\beta}
$$

The term "sine-algebra" comes from the form of the structure constants of $\mathrm{gl}(N)$ Lie algebra, which are given as follows

$$
\left[T_{\alpha}, T_{\beta}\right]=C_{\alpha \beta} T_{\alpha+\beta}, \quad C_{\alpha \beta}=\kappa_{\alpha, \beta}-\kappa_{\beta, \alpha}=2 \imath \sin \left(\frac{\pi \imath}{N}\left(\beta_{1} \alpha_{2}-\beta_{2} \alpha_{1}\right)\right) .
$$

In what follows we denote the index $(0,0)$ as zero for brevity, i.e.

$$
T_{(0,0)}=1_{N}=T_{0}
$$

Relativistic top in GL(2) case was, in fact, described by Sklyanin in [17] as Hamiltonian system with the quadratic Poisson bracket (the classical Sklyanin algebra) through the quasiclassical limit of the quantum exchange (or $R L L-$ ) relations. Here we use the description of $\mathrm{GL}(N)$ top, proposed in papers [10, 11].

Dynamical variables are the components $S_{\alpha}$ of the matrix $S \in \operatorname{Mat}(N, \mathbb{C})$ in the basis (3.1). Let us define the action of the linear operator (it is the multi-dimensional analogue of the inverse inertia tensor in principle axes):

$$
J^{\eta}(S)=\sum_{\alpha \neq 0} T_{\alpha} S_{\alpha} J_{\alpha}^{\eta}=\sum_{\alpha \neq 0} T_{\alpha} S_{\alpha}\left(E_{1}\left(\omega_{\alpha}+\eta\right)-E_{1}\left(\omega_{\alpha}\right)\right), \quad S=\sum_{\alpha} T_{\alpha} S_{\alpha},
$$

where $\omega_{\alpha}$ is defined as in (A.9). Equations of motion are the Euler-Arnold equations for the dynamics of the rigid body in multi-dimensional space:

$$
\dot{S}=\left[S, J^{\eta}(S)\right]
$$

The Lax pair is of the form:

$$
L(z)=\sum_{\alpha} T_{\alpha} S_{\alpha} \varphi_{\alpha}\left(z, \eta+\omega_{\alpha}\right), \quad M(z)=-\sum_{\alpha \neq 0} T_{\alpha} S_{\alpha} \varphi_{\alpha}\left(z, \omega_{\alpha}\right)
$$


Proposition 3.1 The Lax equation (1.4) for the pair of matrices (3.10) is equivalent to equation of motion (3.9).

$\underline{\text { Proof. }}$ The 1.h.s. of the Lax equation is equal to $\sum_{\alpha} T_{\alpha} \dot{S}_{\alpha} \varphi_{\alpha}\left(z, \eta+\omega_{\alpha}\right)$. For the r.h.s. we have

$$
-\sum_{\beta, \gamma \neq 0}\left[T_{\beta}, T_{\gamma}\right] S_{\beta} S_{\gamma} \varphi_{\beta}\left(z, \omega_{\beta}+\eta\right) \varphi_{\gamma}\left(z, \omega_{\gamma}\right),
$$

where the term with $\beta=0$ is absent due to (3.7): it is proportional to the identity matrix. Antisymmetrizing this expression with respect to indices $\beta$ and $\gamma$ we get

$$
\begin{aligned}
& -\frac{1}{2} \sum_{\beta, \gamma \neq 0}\left[T_{\beta}, T_{\gamma}\right] S_{\beta} S_{\gamma}\left(\varphi_{\beta}\left(z, \omega_{\beta}+\eta\right) \varphi_{\gamma}\left(z, \omega_{\gamma}\right)-\varphi_{\gamma}\left(z, \omega_{\gamma}+\eta\right) \varphi_{\beta}\left(z, \omega_{\beta}\right)\right) \underline{A .11], 03.6} \\
& =-\frac{1}{2} \sum_{\beta, \gamma \neq 0} C_{\beta, \gamma} T_{\beta+\gamma} S_{\beta} S_{\gamma} \varphi_{\beta+\gamma}\left(z, \eta+\omega_{\beta+\gamma}\right)\left(J_{\beta}^{\eta}-J_{\gamma}^{\eta}\right)= \\
& =\sum_{\beta, \gamma \neq 0} C_{\beta, \gamma} T_{\beta+\gamma} S_{\beta} S_{\gamma} \varphi_{\beta+\gamma}\left(z, \eta+\omega_{\beta+\gamma}\right) J_{\gamma}^{\eta} .
\end{aligned}
$$

In the basis components (3.1) the equations of motion take the form

$$
\dot{S}_{0}=0, \quad \dot{S}_{\alpha}=\sum_{\beta \neq 0} C_{\beta, \alpha-\beta} S_{\beta} S_{\alpha-\beta} J_{\alpha-\beta}^{\eta}, \quad \alpha \neq 0
$$

which means that the Lax equation is equivalent to (3.9).

The non-relativistic limit $\eta \rightarrow 0$ is taken together with the rescaling of the time variable $t \rightarrow t / \eta$. Equation of motion (3.9) turns into Euler-Arnold equation of the non-relativistic elliptic top [9]:

$$
\dot{S}=[S, J(S)], \quad J(S)=-\sum_{\alpha \neq 0} T_{\alpha} S_{\alpha} E_{2}\left(\omega_{\alpha}\right), \quad S=\sum_{\alpha} T_{\alpha} S_{\alpha} \in \operatorname{Mat}(N, \mathbb{C}),
$$

where the function $E_{2}(\underline{A .3})$ is used.

\section{$4 \mathrm{GL}(N M)$ generalization of spin $M$-body Ruijsenaars-Schneider model}

In this Section we define a generalization of the spin Ruijsenaars-Schneider model from Section 2 and simultaneously for the relativistic top from Section 3. The Lax representation for new model is of size $N M \times N M$. It possesses natural block-matrix structure:

$$
\mathcal{L}(z)=\left(\begin{array}{cccc}
\mathcal{L}^{11}(z) & \mathcal{L}^{12}(z) & \ldots & \mathcal{L}^{1 M}(z) \\
& & & \\
\mathcal{L}^{21}(z) & \mathcal{L}^{22}(z) & \ldots & \mathcal{L}^{2 M}(z) \\
\vdots & \vdots & \ddots & \vdots \\
\mathcal{L}^{M 1}(z) & \mathcal{L}^{M 2}(z) & \ldots & \mathcal{L}^{M M}(z)
\end{array}\right) \quad\left\{\begin{array}{c} 
\\
\text { in each column } \\
M \text { blocks } \\
\text { of size } N \times N
\end{array}\right.
$$


Put it differently,

$$
\mathcal{L}(z)=\sum_{i, j=1}^{M} E_{i j} \otimes \mathcal{L}^{i j}(z) \in \operatorname{Mat}(N M, \mathbb{C}), \quad \mathcal{L}^{i j}(z) \in \operatorname{Mat}(N, \mathbb{C}) .
$$

Likewise the residue of the Lax matrix (1.1) was equal to the matrix $S$, here the residue of the Lax matrix (4.1) is equal to $\operatorname{Res}_{z=0} \mathcal{L}(z)=\mathcal{S} \in \operatorname{Mat}(N M, \mathbb{C})$, and for each block we have

$$
\mathcal{L}^{i j}(z)=\mathcal{L}^{i j}\left(\mathcal{S}^{i j}, z\right), \quad \mathcal{S}^{i j}=\operatorname{Res}_{z=0} \mathcal{L}^{i j}(z) \in \operatorname{Mat}(N, \mathbb{C})
$$

Explicit expression for the $i j$-th block of the Lax matrix takes the form

$$
\mathcal{L}^{i j}(z)=\sum_{\alpha} T_{\alpha} \mathcal{S}_{\alpha}^{i j} \varphi_{\alpha}\left(z, \omega_{\alpha}+q_{i j}+\eta\right), \quad q_{i j}=q_{i}-q_{j}, \quad \omega_{\alpha}=\frac{\alpha_{1}+\alpha_{2} \tau}{N}
$$

and for $i j$-th block of the accompany $M$-matrix we get

$$
\begin{gathered}
\mathcal{M}^{i j}(z)=-\sum_{\alpha} T_{\alpha} \mathcal{S}_{\alpha}^{i j} \varphi_{\alpha}\left(z, \omega_{\alpha}+q_{i j}\right), \quad \text { for } i \neq j \\
\mathcal{M}^{i i}(z)=-T_{0} \mathcal{S}_{0}^{i i}\left(E_{1}(z)+E_{1}(\eta)\right)-\sum_{\alpha \neq 0} T_{\alpha} \mathcal{S}_{\alpha}^{i i} \varphi_{\alpha}\left(z, \omega_{\alpha}\right) .
\end{gathered}
$$

In the case $N=1$ we are left with the scalar parts of the above matrices only, i.e. with those proportional to $T_{0}=1_{N}$. In this way the Lax pair (1.1)-(1.2) is reproduced. When $M=1$ we are left with a single (diagonal) block. In this case we get the Lax pair (3.10) up to the term $-E_{1}(\eta) T_{0} S_{0}^{11}$ in the $M$-matrix. But for $M=1$ it does not effect the equations of motion since it is proportional to the identity matrix.

To have a compact form of equations of motion let us define the following linear operator, acting on $i j$-th block (when $i \neq j$ ) of the matrix $\mathcal{S}$ :

$$
J^{\eta, q_{i j}}\left(\mathcal{S}^{i j}\right)=\sum_{\alpha} T_{\alpha} \mathcal{S}_{\alpha}^{i j}\left(E_{1}\left(\omega_{\alpha}+q_{i j}+\eta\right)-E_{1}\left(\omega_{\alpha}+q_{i j}\right)\right) .
$$

At the same time for the diagonal blocks we use the linear operator $J^{\eta}(3.8)$. As will be shown below, the equations of motion coming from the Lax equation with the Lax pair (4.4)-(4.5) take the following form for the diagonal blocks of the matrix $\mathcal{S}$ :

$$
\dot{\mathcal{S}}^{i i}=\left[\mathcal{S}^{i i}, J^{\eta}\left(\mathcal{S}^{i i}\right)\right]+\sum_{k: k \neq i}^{M}\left(\mathcal{S}^{i k} J^{\eta, q_{k i}}\left(S^{k i}\right)-J^{\eta, q_{i k}}\left(\mathcal{S}^{i k}\right) S^{k i}\right),
$$

and for the non-diagonal blocks we get

$$
\begin{gathered}
\dot{\mathcal{S}}^{i j}=\mathcal{S}^{i j} J^{\eta}\left(\mathcal{S}^{j j}\right)-J^{\eta}\left(\mathcal{S}^{i i}\right) \mathcal{S}^{i j}+\mathcal{S}^{i i} J^{\eta, q_{i j}}\left(\mathcal{S}^{i j}\right)-J^{\eta, q_{i j}}\left(\mathcal{S}^{i j}\right) \mathcal{S}^{j j}+ \\
+\sum_{k: k \neq i, j}^{M}\left(\mathcal{S}^{i k} J^{\eta, q_{k j}}\left(\mathcal{S}^{k j}\right)-J^{\eta, q_{i k}}\left(\mathcal{S}^{i k}\right) \mathcal{S}^{k j}\right)
\end{gathered}
$$


or, equivalently,

$$
\dot{\mathcal{S}}^{i j}=\mathcal{S}^{i j} J^{\eta}\left(\mathcal{S}^{j j}\right)-J^{\eta}\left(\mathcal{S}^{i i}\right) \mathcal{S}^{i j}+\sum_{k: k \neq j}^{M} \mathcal{S}^{i k} J^{\eta, q_{k j}}\left(\mathcal{S}^{k j}\right)-\sum_{k: k \neq i}^{M} J^{\eta, q_{i k}}\left(\mathcal{S}^{i k}\right) \mathcal{S}^{k j} .
$$

For the positions of particles the following equation holds

$$
\ddot{q}_{i}=\frac{1}{N} \operatorname{tr}\left(\dot{\mathcal{S}}^{i i}\right)=\frac{1}{N} \sum_{k: k \neq i}^{M} \operatorname{tr}\left(\mathcal{S}^{i k} J^{\eta, q_{k i}}\left(\mathcal{S}^{k i}\right)-J^{\eta, q_{i k}}\left(\mathcal{S}^{i k}\right) \mathcal{S}^{k i}\right),
$$

which is deduced from equation (4.7) by taking the trace of both parts together with the conditions

$$
\dot{q}_{i}=\mathcal{S}_{0}^{i i}=\frac{1}{N} \operatorname{tr}\left(\mathcal{S}^{i i}\right), \quad i=1, \ldots, M,
$$

- analogues of relations (1.3) for the generalized model.

Notice that for $N=1$ the linear operators $J^{\eta, q_{i j}}\left(\mathcal{S}^{i j}\right)$ (4.6) and $J^{\eta}\left(\mathcal{S}^{i i}\right)$ (3.8) take the form

$$
N=1: \quad J^{\eta, q_{i j}}\left(S_{i j}\right)=\left(E_{1}\left(q_{i j}+\eta\right)-E_{1}\left(q_{i j}\right)\right) S_{i j}, \quad J^{\eta}\left(S_{i i}\right)=0 .
$$

The operator $J^{\eta}$ is equal to zero in the case $N=1$ due to the absence of the scalar term (with $\alpha=0$ ) in the r.h.s. of equation (3.8). Using (4.12), it is easy to see that for $N=1$ the equations of motion (4.7)-(4.9) and (4.10) turn into (1.5)-(1.7) for $M$-body spin RuijsenaarsSchneider model. Let us prove the statement similar to the Proposition 2.1.

Proposition 4.1 Equations of motion $\mathcal{S}$ (4.7), (4.8)-(4.9) for diagonal and off-diagonal blocks of matrix $\mathcal{S}$ are equivalent to the Lax equation with additional term

$$
\dot{\mathcal{L}}(z)=[\mathcal{L}(z), \mathcal{M}(z)]+\sum_{i, j=1}^{M} \sum_{\alpha} E_{i j} \otimes T_{\alpha}\left(\mu_{0}^{i}-\mu_{0}^{j}\right) \mathcal{S}_{\alpha}^{i j} f_{\alpha}\left(z, \omega_{\alpha}+q_{i j}+\eta\right)
$$

for the Lax pair (4.4)-(4.5) and the set of variables

$$
\mu_{0}^{i}=\dot{q}_{i}-\mathcal{S}_{0}^{i i}=\dot{q}_{i}-\frac{1}{N} \operatorname{tr}\left(\mathcal{S}^{i i}\right), \quad i=1, \ldots, M .
$$

On-shell constraints $\mu_{0}^{i}=0$ the matrices $(4.4)$ - 4.5$)$ satisfy the Lax equation $\dot{\mathcal{L}}(z)=[\mathcal{L}(z), \mathcal{M}(z)]$ and provide equations of motion (4.7)-(4.9) and (4.10).

Proof. The proof is similar to the one for Proposition 2.1. Consider the l.h.s. of (4.13):

$$
\dot{\mathcal{L}^{i i}}(z)=\sum_{\alpha} T_{\alpha} \dot{\mathcal{S}}_{\alpha}^{i i} \varphi_{\alpha}\left(z, \omega_{\alpha}+\eta\right)
$$

For $i \neq j$ we have

$$
i \neq j: \quad \dot{\mathcal{L}^{i j}}(z)=\sum_{\alpha} T_{\alpha}\left(\dot{\mathcal{S}}_{\alpha}^{i j} \varphi_{\alpha}\left(z, \omega_{\alpha}+q_{i j}+\eta\right)+\left(\dot{q}_{i}-\dot{q}_{j}\right) \mathcal{S}_{\alpha}^{i j} f_{\alpha}\left(z, \omega_{\alpha}+q_{i j}+\eta\right)\right)=
$$




$$
\begin{aligned}
& =\sum_{\alpha} T_{\alpha} \dot{\mathcal{S}}_{\alpha}^{i j} \varphi_{\alpha}\left(z, \omega_{\alpha}++q_{i j}+\eta\right)+\sum_{\alpha} T_{\alpha}\left(\mu_{0}^{i}-\mu_{0}^{j}\right) \mathcal{S}_{\alpha}^{i j} f_{\alpha}\left(z, \omega_{\alpha}+q_{i j}+\eta\right)+ \\
& +\sum_{\alpha} T_{\alpha}\left(\mathcal{S}_{0}^{i i}-\mathcal{S}_{0}^{j j}\right) \mathcal{S}_{\alpha}^{i j} \varphi_{\alpha}\left(z, \omega_{\alpha}+q_{i j}+\eta\right)\left(E_{1}\left(z+\omega_{\alpha}+q_{i j}+\eta\right)-E_{1}\left(\omega_{\alpha}+q_{i j}+\eta\right)\right) .
\end{aligned}
$$

The expression (function) in the last sum in the r.h.s. is just the function $f_{\alpha}\left(z, \omega_{\alpha}+q_{i j}+\eta\right)$ from (A.10), being transformed through (A.8). The second sum in the r.h.s. of (4.16) exactly cancels out with the additional term from the r.h.s. of equation (4.13). The rest of the terms from the r.h.s. (i.e. those coming from the commutator) are of the following form for the diagonal block

$$
\left[\mathcal{L}^{i i}, \mathcal{M}^{i i}\right]+\sum_{k: k \neq i} \mathcal{L}^{i k} \mathcal{M}^{k i}-\mathcal{M}^{i k} \mathcal{L}^{k i}
$$

and for the off-diagonal $i j$-th block $(i \neq j)$ - the rest of the terms are

$$
i \neq j: \quad \mathcal{L}^{i i} \mathcal{M}^{i j}-\mathcal{M}^{i i} \mathcal{L}^{i j}+\mathcal{L}^{i j} \mathcal{M}^{j j}-\mathcal{M}^{i j} \mathcal{L}^{j j}+\sum_{k: k \neq i, j} \mathcal{L}^{i k} \mathcal{M}^{k j}-\mathcal{M}^{i k} \mathcal{L}^{k j}
$$

The computations for the diagonal blocks are very similar to those performed in Sections 2 and 3. The first term (commutator) in (4.17) provides the first term (also a commutator) in the r.h.s. of the equation of motion (4.7) in the same way as it was made for the relativistic top for the equation (3.9). The sum in (4.17) is simplified similarly to (2.4):

$$
\begin{gathered}
\mathcal{L}^{i k} \mathcal{M}^{k i}-\mathcal{M}^{i k} \mathcal{L}^{k i}=\sum_{\beta, \gamma} T_{\beta} T_{\gamma} \mathcal{S}_{\beta}^{i k} \mathcal{S}_{\gamma}^{k i} \times \\
\times\left(\varphi_{\beta}\left(z, \omega_{\beta}+q_{i k}\right) \varphi_{\gamma}\left(z, \omega_{\gamma}+q_{k i}+\eta\right)-\varphi_{\beta}\left(z, \omega_{\beta}+q_{i k}+\eta\right) \varphi_{\gamma}\left(z, \omega_{\gamma}+q_{k i}\right)\right) .
\end{gathered}
$$

Applying (A.11) to the expression in the brackets we get

$$
\begin{gathered}
\sum_{\beta, \gamma} T_{\beta} T_{\gamma} \mathcal{S}_{\beta}^{i k} \mathcal{S}_{\gamma}^{k i} \varphi_{\beta+\gamma}\left(z, \omega_{\beta+\gamma}+q_{i i}+\eta\right)\left(E_{1}\left(\omega_{\gamma}+q_{k i}+\eta\right)-\right. \\
\left.-E_{1}\left(\omega_{\gamma}+q_{k i}\right)-E_{1}\left(\omega_{\beta}+q_{i k}+\eta\right)+E_{1}\left(\omega_{\beta}+q_{i k}\right)\right),
\end{gathered}
$$

which yields the sum in the r.h.s. of equations of motion (4.7). The scalar (i.e. zero) component, which provides (4.11), corresponds to the terms with $\beta=-\gamma$ due to relations (3.4)-(3.5).

Consider the off-diagonal block $i j$. For the first four terms from (4.18) let us write down separately all the summands containing the scalar (zero) components of the diagonal blocks of matrix $\mathcal{S}$ (i.e. the summands containing $\mathcal{S}_{0}^{i i}$ ):

$$
\begin{gathered}
\left(\mathcal{S}_{0}^{i i}-\mathcal{S}_{0}^{j j}\right) \sum_{\alpha} T_{\alpha} \mathcal{S}_{\alpha}^{i j}\left(\left(E_{1}(z)+E_{1}(\eta)\right) \varphi_{\alpha}\left(z, \omega_{\alpha}+q_{i j}+\eta\right)-\varphi_{\alpha}\left(z, \omega_{\alpha}+q_{i j}\right) \phi(z, \eta)\right) \\
\stackrel{\text { A.11 }}{=}\left(\mathcal{S}_{0}^{i i}-\mathcal{S}_{0}^{j j}\right) \sum_{\alpha} T_{\alpha} \mathcal{S}_{\alpha}^{i j} \varphi_{\alpha}\left(z, \omega_{\alpha}+q_{i j}+\eta\right)\left(E_{1}\left(z+\omega_{\alpha}+q_{i j}+\eta\right)-E_{1}\left(\omega_{\alpha}+q_{i j}\right)\right) .
\end{gathered}
$$

There are the same type terms (containing $\mathcal{S}_{0}^{i i}$ ) in the l.h.s. of equation (4.13). They are in the last sum in (4.16). Transpose them to the r.h.s. and sum up with the result (4.21). Then we get

$$
\left(\mathcal{S}_{0}^{i i}-\mathcal{S}_{0}^{j j}\right) \sum_{\alpha} T_{\alpha} \mathcal{S}_{\alpha}^{i j} \varphi_{\alpha}\left(z, \omega_{\alpha}+q_{i j}+\eta\right)\left(E_{1}\left(\omega_{\alpha}+q_{i j}+\eta\right)-E_{1}\left(\omega_{\alpha}+q_{i j}\right)\right)
$$


Next, write down the rest of the first four terms in (4.18), i.e. the terms, which do not contain $\mathcal{S}_{0}^{i i}$ :

$$
\begin{gathered}
\sum^{\prime} T_{\beta} T_{\gamma} \mathcal{S}_{\beta}^{i i} \mathcal{S}_{\gamma}^{i j}\left(\varphi_{\beta}\left(z, \omega_{\beta}\right) \varphi_{\gamma}\left(z, \omega_{\gamma}+q_{i j}+\eta\right)-\varphi_{\beta}\left(z, \omega_{\beta}+\eta\right) \varphi_{\gamma}\left(z, \omega_{\gamma}+q_{i j}\right)\right) \\
-\sum^{\prime} T_{\gamma} T_{\beta} \mathcal{S}_{\gamma}^{i j} \mathcal{S}_{\beta}^{j j}\left(\varphi_{\beta}\left(z, \omega_{\beta}\right) \varphi_{\gamma}\left(z, \omega_{\gamma}+q_{i j}+\eta\right)-\varphi_{\beta}\left(z, \omega_{\beta}+\eta\right) \varphi_{\gamma}\left(z, \omega_{\gamma}+q_{i j}\right)\right),
\end{gathered}
$$

where the primed sum $\sum^{\prime}-$ is the sum over two indices $\beta, \gamma \in \mathbb{Z}_{N}^{\times 2}$ with condition $\beta \neq 0$ (the terms with $\beta=0$ were already accounted in (4.21) ). Applying (A.11), we obtain

$$
\begin{aligned}
& =\sum^{\prime} T_{\gamma} T_{\beta} \mathcal{S}_{\gamma}^{i j} \mathcal{S}_{\beta}^{j j} \varphi_{\beta+\gamma}\left(z, \omega_{\beta+\gamma}+q_{i j}+\eta\right)\left(E_{1}\left(\omega_{\beta}+\eta\right)-E_{1}\left(\omega_{\beta}\right)\right)- \\
& -\sum^{\prime} T_{\beta} T_{\gamma} \mathcal{S}_{\beta}^{i i} \mathcal{S}_{\gamma}^{i j} \varphi_{\beta+\gamma}\left(z, \omega_{\beta+\gamma}+q_{i j}+\eta\right)\left(E_{1}\left(\omega_{\beta}+\eta\right)-E_{1}\left(\omega_{\beta}\right)\right)+ \\
& +\sum^{\prime} T_{\beta} T_{\gamma} \mathcal{S}_{\beta}^{i i} \mathcal{S}_{\gamma}^{i j} \varphi_{\beta+\gamma}\left(z, \omega_{\beta+\gamma}+q_{i j}+\eta\right)\left(E_{1}\left(\omega_{\gamma}+q_{i j}+\eta\right)-E_{1}\left(\omega_{\gamma}+q_{i j}\right)\right)- \\
& -\sum^{\prime} T_{\gamma} T_{\beta} \mathcal{S}_{\gamma}^{i j} \mathcal{S}_{\beta}^{j j} \varphi_{\beta+\gamma}\left(z, \omega_{\beta+\gamma}+q_{i j}+\eta\right)\left(E_{1}\left(\omega_{\gamma}+q_{i j}+\eta\right)-E_{1}\left(\omega_{\gamma}+q_{i j}\right)\right) .
\end{aligned}
$$

Thus, in the r.h.s. of the Lax equation we are left with the terms (4.24) and (4.22). Notice that addition of the term (4.22) to (4.24) is equivalent to removing the primes in the last two sums in (4.24) since the terms (4.22) correspond to the index value $\beta=0$ in these sums. Therefore, the sum of expressions (4.22) and (4.24) reproduces the first four terms from the r.h.s. of equation of motion (4.8).

It remains to determine the contribution to equation of motion coming from the last sum in (4.18). For this purpose consider the difference

$$
\begin{gathered}
\mathcal{L}^{i k} \mathcal{M}^{k j}-\mathcal{M}^{i k} \mathcal{L}^{k j}=\sum_{\beta, \gamma} T_{\beta} T_{\gamma} \mathcal{S}_{\beta}^{i k} \mathcal{S}_{\gamma}^{k j} \times \\
\times\left(\varphi_{\beta}\left(z, \omega_{\beta}+q_{i k}\right) \varphi_{\gamma}\left(z, \omega_{\gamma}+q_{k j}+\eta\right)-\varphi_{\beta}\left(z, \omega_{\beta}+q_{i k}+\eta\right) \varphi_{\gamma}\left(z, \omega_{\gamma}+q_{k j}\right)\right) .
\end{gathered}
$$

Again, applying (A.11) to the expression in the brackets, we get the answer

$$
\begin{gathered}
\sum_{\beta, \gamma} T_{\beta} T_{\gamma} \mathcal{S}_{\beta}^{i k} \mathcal{S}_{\gamma}^{k j} \varphi_{\beta+\gamma}\left(z, \omega_{\beta+\gamma}+q_{i j}+\eta\right)\left(E_{1}\left(\omega_{\gamma}+q_{k j}+\eta\right)-\right. \\
\left.-E_{1}\left(\omega_{\gamma}+q_{k j}\right)-E_{1}\left(\omega_{\beta}+q_{i k}+\eta\right)+E_{1}\left(\omega_{\beta}+q_{i k}\right)\right),
\end{gathered}
$$

which yields the sum from the r.h.s. of equation of motion (4.8). This finishes the proof.

Similarly to (2.8)-(2.12) and (3.14) in the non-relativistic limit $\eta \rightarrow 0$ we have

$$
\begin{gathered}
\dot{\mathcal{S}}^{i i}=\left[\mathcal{S}^{i i}, J\left(\mathcal{S}^{i i}\right)\right]+\sum_{k: k \neq i}^{M}\left(\mathcal{S}^{i k} J^{q_{k i}}\left(S^{k i}\right)-J^{q_{i k}}\left(\mathcal{S}^{i k}\right) S^{k i}\right), \\
\dot{\mathcal{S}}^{i j}=\mathcal{S}^{i j} J\left(\mathcal{S}^{j j}\right)-J\left(\mathcal{S}^{i i}\right) \mathcal{S}^{i j}+\sum_{k: k \neq j}^{M} \mathcal{S}^{i k} J^{q_{k j}}\left(\mathcal{S}^{k j}\right)-\sum_{k: k \neq i}^{M} J^{q_{i k}}\left(\mathcal{S}^{i k}\right) \mathcal{S}^{k j},
\end{gathered}
$$


and

$$
\ddot{q}_{i}=-\frac{1}{N} \sum_{k: k \neq i}^{M} \partial_{q_{i}} \operatorname{tr}\left(J^{q_{i k}}\left(\mathcal{S}^{i k}\right) \mathcal{S}^{k i}\right),
$$

where $J\left(\mathcal{S}^{j j}\right)$ are as in (3.14), and

$$
J^{q_{i j}}\left(\mathcal{S}^{i j}\right)=-\sum_{\alpha} T_{\alpha} \mathcal{S}_{\alpha}^{i j} E_{2}\left(\omega_{\alpha}+q_{i j}\right)
$$

The last equation of motion (4.29) is obtained similarly to (2.9). That is for the operator $J^{\eta, q_{i j}}\left(\mathcal{S}^{i j}\right)$ (4.6) we use the following expansion near $\eta=0$ :

$$
J^{\eta, q_{i j}}\left(\mathcal{S}^{i j}\right)=\eta J^{q_{i j}}\left(\mathcal{S}^{i j}\right)+\frac{1}{2} \eta^{2} \partial_{q_{i}} J^{q_{i j}}\left(\mathcal{S}^{i j}\right)+O\left(\eta^{3}\right)
$$

For $N=1$ equations (4.27)-(4.29) turn into equations (2.11)-(2.12) for the spin Calogero-Moser model.

The equations (4.27)-(4.30) were derived in recent paper $[7]^{2}$ for a more general case. Constraints (4.14) in the non-relativistic limit take the form

$$
\frac{1}{N} \operatorname{tr}\left(\mathcal{S}^{i i}\right)=0, \quad i=1, \ldots, M
$$

The comment made in the end of Section 2, is applicable to these constraints as well. The gauge fixation is performed in this case with respect to the coadjoint action of $M$-dimensional subgroup in the Cartan subgroup of $\mathrm{GL}(N M, \mathbb{C})$, i.e. with respect to conjugation by matrices of the form $D=\sum_{k} d_{k} E_{k k} \otimes T_{0}$.

\section{$5 \quad$ Interacting relativistic tops}

In this Section we consider the special case of the $\mathrm{GL}(N M, \mathbb{C})$ model from the previous Section when the matrix $\mathcal{S}$ is of rank 1, i.e.

$$
\mathcal{S}^{i j}=\xi^{i} \otimes \rho^{j} \in \operatorname{Mat}(N, \mathbb{C}), \quad i, j=1, \ldots, M,
$$

or

$$
\mathcal{S}_{a b}^{i j}=\xi_{a}^{i} \rho_{b}^{j}, \quad i, j=1, \ldots, M, \quad a, b=1, \ldots, N,
$$

where $\xi^{i}$ - is a set of $M$ vector-columns of hight $N$ each, and $\rho^{i}$ - is a set of $M$ vector-rows of length $N$ each.

The purpose of the Section - is to rewrite the r.h.s. of equations of motion (4.7) for the diagonal blocks in terms of the diagonal blocks only. Then for $M$ diagonal blocks we will get a closed system of $M$ matrix equations of motion. A problem in the usage of conditions (5.1)-(5.2) is that they are written in the standard basis, while for the operators $J^{\eta, q_{k i}}$ (4.6) from equations (4.7) the basis $T_{\alpha}$ (3.1) is used. Let us use the tensor notation to overcome this difficulty.

\footnotetext{
${ }^{2}$ In the elliptic case in [7 a slightly different normalization factors are used. In particular, the positions of particles are divided by $N$ everywhere.
} 
Consider an operator $A$ of the form

$$
A(S)=\sum_{\alpha} T_{\alpha} S_{\alpha} A_{\alpha} \in \operatorname{Mat}(N, \mathbb{C}), \quad S=\sum_{\alpha} T_{\alpha} S_{\alpha} \in \operatorname{Mat}(N, \mathbb{C})
$$

and introduce notations

$$
\begin{aligned}
& A_{12}=\sum_{\alpha} A_{\alpha} T_{\alpha} \otimes T_{-\alpha} \in \operatorname{Mat}(N, \mathbb{C})^{\otimes 2} \\
& \breve{A}_{12}=\sum_{\alpha} \breve{A}_{\alpha} T_{\alpha} \otimes T_{-\alpha} \in \operatorname{Mat}(N, \mathbb{C})^{\otimes 2}
\end{aligned}
$$

and

$$
\begin{gathered}
\breve{A}_{12}=A_{12} P_{12}, \\
\breve{A}_{21}=\sum_{\alpha} \breve{A}_{\alpha} T_{-\alpha} \otimes T_{\alpha}=\sum_{\alpha} \breve{A}_{-\alpha} T_{\alpha} \otimes T_{-\alpha}=P_{12} \breve{A}_{12} P_{12}=P_{12} A_{12},
\end{gathered}
$$

where $P_{12}$ - is the permutation operator ${ }^{3}$

$$
P_{12}=\sum_{a, b=1}^{N} e_{a b} \otimes e_{b a}=\frac{1}{N} \sum_{\alpha} T_{\alpha} \otimes T_{-\alpha} .
$$

Let us write down a few main properties of the permutation operator: $\left(P_{12}\right)^{2}=1_{N} \otimes 1_{N}$ and $(B \otimes C) P_{12}=P_{12}(C \otimes B)$ for matrices $B, C \in \operatorname{Mat}(N, \mathbb{C})$. Also, using the standard notations (see e.g. [17]) $S_{1}=S \otimes 1_{N}$ and $S_{2}=1_{N} \otimes S$, we have $S_{2} P_{12}=P_{12} S_{1}$ and

$$
\operatorname{tr}_{2}\left(P_{12} S_{2}\right)=N S_{1}
$$

where $\operatorname{tr}_{2}$ - is a trace over the second tensor component.

Using the above mentioned notations the operator (5.3) takes the form

$$
\begin{gathered}
A(S) \stackrel{3.5}{=} \frac{1}{N} \operatorname{tr}_{2}\left(A_{12} S_{2}\right)=\frac{1}{N} \operatorname{tr}_{2}\left(\breve{A}_{12} P_{12} S_{2}\right)=\frac{1}{N} \operatorname{tr}_{2}\left(S_{2} \breve{A}_{12} P_{12}\right)= \\
=\frac{1}{N} \operatorname{tr}_{2}\left(S_{2} P_{12} \breve{A}_{21}\right)=\frac{1}{N} \operatorname{tr}_{2}\left(P_{12} S_{1} \breve{A}_{21}\right)
\end{gathered}
$$

where in the last equality in the first line we used a cyclic permutation of matrices in the second tensor component.

Consider expression

$$
\mathcal{S}^{i k} A\left(\mathcal{S}^{k i}\right) \stackrel{5.9}{=} \frac{1}{N} \operatorname{tr}_{2}\left(\mathcal{S}_{1}^{i k} P_{12} \mathcal{S}_{1}^{k i} \breve{A}_{21}\right)
$$

For the matrix (5.1) we get

$$
\begin{gathered}
\mathcal{S}_{1}^{i k} P_{12} \mathcal{S}_{1}^{k i}=\sum_{a, b=1}^{N}\left(\left(\xi^{i} \otimes \rho^{k}\right) e_{a b}\left(\xi^{k} \otimes \rho^{i}\right)\right) \otimes e_{b a}=\sum_{a, b=1}^{N}\left(\xi^{i} \otimes \rho^{i}\right)\left(\rho^{k} e_{a b} \xi^{k}\right) \otimes e_{b a}= \\
=\sum_{a, b=1}^{N} \mathcal{S}^{i i} \operatorname{tr}\left(e_{a b} \mathcal{S}^{k k}\right) \otimes e_{b a}=\mathcal{S}_{1}^{i i} \mathcal{S}_{2}^{k k},
\end{gathered}
$$

\footnotetext{
${ }^{3}$ The set of matrices $\left\{e_{a b}\right\}$ in (5.7) forms the standard basis of the $\operatorname{space} \operatorname{Mat}(N, \mathbb{C})$.
} 
where we used that $\left(\rho^{k} e_{a b} \xi^{k}\right)$ is a scalar. Then for (5.10) we have

$$
\mathcal{S}^{i k} A\left(\mathcal{S}^{k i}\right)=\frac{1}{N} \mathcal{S}^{i i} \operatorname{tr}_{2}\left(\breve{A}_{21} \mathcal{S}_{2}^{k k}\right)
$$

Similarly,

$$
A\left(\mathcal{S}^{i k}\right) \mathcal{S}^{k i}=\frac{1}{N} \operatorname{tr}_{2}\left(\breve{A}_{12} \mathcal{S}_{1}^{i k} P_{12} \mathcal{S}_{1}^{k i}\right)=\frac{1}{N} \operatorname{tr}_{2}\left(\breve{A}_{12} \mathcal{S}_{2}^{k k}\right) \mathcal{S}^{i i} .
$$

By applying (5.12) and (5.13) to equation (4.10), we get the following statement.

Proposition 5.1 In the case (5.1) when the matrix of spin variables is of rank one the equations of motion (4.7) take the following form

$$
\begin{gathered}
\dot{\mathcal{S}}^{i i}=\left[\mathcal{S}^{i i}, J^{\eta}\left(\mathcal{S}^{i i}\right)\right]+\sum_{k: k \neq i}^{M}\left(\mathcal{S}^{i i} \widetilde{J}^{\eta, q_{k i}}\left(S^{k k}\right)-\breve{J}^{\eta, q_{i k}}\left(\mathcal{S}^{k k}\right) S^{i i}\right), \\
\ddot{q}_{i}=\frac{1}{N} \operatorname{tr}\left(\dot{\mathcal{S}}^{i i}\right)=\frac{1}{N} \sum_{k: k \neq i}^{M} \operatorname{tr}\left(\mathcal{S}^{i i} \widetilde{J}^{\eta, q_{k i}}\left(S^{k k}\right)-\breve{J}^{\eta, q_{i k}}\left(\mathcal{S}^{k k}\right) S^{i i}\right),
\end{gathered}
$$

whert $4^{4}$

$$
\begin{aligned}
\widetilde{J}^{\eta, q_{i j}}\left(\mathcal{S}^{k k}\right) & =\frac{1}{N} \operatorname{tr}_{2}\left(\breve{J}_{21}^{\eta, q_{i j}} \mathcal{S}_{2}^{k k}\right) \\
\breve{J}^{\eta, q_{i j}}\left(\mathcal{S}^{k k}\right) & =\frac{1}{N} \operatorname{tr}_{2}\left(\breve{J}_{12}^{\eta, q_{i j}} \mathcal{S}_{2}^{k k}\right)
\end{aligned}
$$

and

$$
J_{12}^{\eta, q_{i j}}=\sum_{\alpha} T_{\alpha} \otimes T_{-\alpha}\left(E_{1}\left(\omega_{\alpha}+q_{i j}+\eta\right)-E_{1}\left(\omega_{\alpha}+q_{i j}\right)\right)
$$

Let us derive explicit expression for $\breve{J}_{12}^{\eta, q_{i j}}=J_{12}^{\eta, q_{i j}} P_{12}$ from (5.16) -(5.17):

$$
\begin{aligned}
& J_{12}^{\eta, q_{i j}} P_{12}=\frac{1}{N} \sum_{\alpha, \gamma} T_{\alpha} T_{\gamma} \otimes T_{-\alpha} T_{-\gamma}\left(E_{1}\left(\omega_{\alpha}+q_{i j}+\eta\right)-E_{1}\left(\omega_{\alpha}+q_{i j}\right)\right) \stackrel{(3.4)}{=} \\
& =\frac{1}{N} \sum_{\alpha, \gamma} \kappa_{\alpha, \gamma}^{2} T_{\alpha+\gamma} \otimes T_{-\alpha-\gamma}\left(E_{1}\left(\omega_{\alpha}+q_{i j}+\eta\right)-E_{1}\left(\omega_{\alpha}+q_{i j}\right)\right)= \\
& =\frac{1}{N} \sum_{\gamma} T_{\gamma} \otimes T_{-\gamma} \sum_{\alpha} \kappa_{\alpha, \gamma}^{2}\left(E_{1}\left(\omega_{\alpha}+q_{i j}+\eta\right)-E_{1}\left(\omega_{\alpha}+q_{i j}\right)\right) .
\end{aligned}
$$

The latter sum over index $\alpha$ is the finite-dimensional Fourier transformation of the expression in the brackets. Let us use the formulae (see [18]):

$$
\frac{1}{N} \sum_{\alpha}\left(E_{1}\left(\omega_{\alpha}+\eta\right)+2 \pi \imath \partial_{\tau} \omega_{\alpha}\right)=E_{1}(N \eta)
$$

and

$$
\frac{1}{N} \sum_{\alpha} \kappa_{\alpha, \gamma}^{2}\left(E_{1}\left(\omega_{\alpha}+\eta\right)+2 \pi \imath \partial_{\tau} \omega_{\alpha}\right)=\varphi_{\gamma}\left(N \eta, \omega_{\gamma}\right), \quad \text { for } \gamma \neq 0
$$

\footnotetext{
${ }^{4}$ Explicit expressions for $\tilde{J}^{\eta, q_{i j}}, \breve{J}^{\eta, q_{i j}}$ (5.16)-(5.17) are given below in (5.24) (5.25).
} 
Plugging it into (5.19), we get

$$
\breve{J}_{12}^{\eta, q_{i j}}=J_{12}^{\eta, q_{i j}} P_{12}=\sum_{\alpha} I_{\alpha}^{\eta, q_{i j}} T_{\alpha} \otimes T_{-\alpha}
$$

where

$$
\begin{gathered}
I_{0}^{\eta, q_{i j}}=E_{1}\left(N q_{i j}+N \eta\right)-E_{1}\left(N q_{i j}\right), \\
I_{\alpha}^{\eta, q_{i j}}=\varphi_{\alpha}\left(N q_{i j}+N \eta, \omega_{\alpha}\right)-\varphi_{\alpha}\left(N q_{i j}, \omega_{\alpha}\right), \quad \alpha \neq 0 .
\end{gathered}
$$

In this way we also get explicit answer for (5.16)-(5.17):

$$
\breve{J}^{\eta, q_{i j}}\left(\mathcal{S}^{k k}\right)=\frac{1}{N} \operatorname{tr}_{2}\left(\breve{J}_{12}^{\eta, q_{i j}} \mathcal{S}_{2}^{k k}\right)=\sum_{\alpha} T_{\alpha} \mathcal{S}_{\alpha}^{k k} I_{\alpha}^{\eta, q_{i j}}
$$

and

$$
\widetilde{J}^{\eta, q_{i j}}\left(\mathcal{S}^{k k}\right)=\frac{1}{N} \operatorname{tr}_{2}\left(\breve{J}_{21}^{\eta, q_{i j}} \mathcal{S}_{2}^{k k}\right)=\sum_{\alpha} T_{\alpha} \mathcal{S}_{\alpha}^{k k} I_{-\alpha}^{\eta, q_{i j}} .
$$

In the non-relativistic limit $\eta \rightarrow 0(2.8)$ we have

$$
\begin{gathered}
\dot{\mathcal{S}}^{i i}=\left[\mathcal{S}^{i i}, J\left(\mathcal{S}^{i i}\right)\right]+\sum_{k: k \neq i}^{M}\left[\mathcal{S}^{i i}, \breve{J}^{q_{i k}}\left(\mathcal{S}^{k k}\right)\right], \\
\ddot{q}_{i}=-\frac{1}{N} \sum_{k: k \neq i}^{M} \partial_{q_{i}} \operatorname{tr}\left(\mathcal{S}^{i i} \breve{J}^{q_{i k}}\left(\mathcal{S}^{k k}\right)\right),
\end{gathered}
$$

where

$$
\breve{J}^{q_{i j}}\left(\mathcal{S}^{k k}\right)=\left.\partial_{\eta} \breve{J}^{\eta, q_{i j}}\left(\mathcal{S}^{k k}\right)\right|_{\eta=0}=\partial_{q_{i}} \sum_{\alpha} T_{\alpha} \mathcal{S}_{\alpha}^{k k} F_{\alpha}^{q_{i j}}
$$

and

$$
F_{0}^{q_{i j}}=E_{1}\left(N q_{i j}\right), \quad F_{\alpha}^{q_{i j}}=\varphi_{\alpha}\left(N q_{i j}, \omega_{\alpha}\right) .
$$

Such answer follows due to evenness of the function $E_{2}(z)$. This leads to the properties:

$$
\left.\partial_{\eta} \breve{J}_{21}^{\eta, q}\right|_{\eta=0}=\left.\partial_{\eta} \breve{J}_{12}^{\eta,-q}\right|_{\eta=0}=\left.\partial_{\eta} \widetilde{J}_{12}^{\eta,-q}\right|_{\eta=0}=\left.\partial_{\eta} \widetilde{J}_{21}^{\eta, q}\right|_{\eta=0}
$$

The classical spin variables in the models of Calogero and Ruijsenaars types are often described in terms of quiver parametrization [3, 1, 13, 4]. For $\operatorname{GL}(M, \mathbb{C})$ model the latter means introducing $2 N M$ variables $\xi_{a}^{i}, \rho_{a}^{i}, i=1, \ldots, M, a=1, \ldots, N$, so that the spin variables (in $\mathrm{GL}(M, \mathbb{C})$ case) are of the form $S_{i j}=\sum_{a} \xi_{a}^{i} \rho_{a}^{j}$. In the trigonometric and rational cases the Poisson structure is known, and one can write equations of motion for the set of $\operatorname{Mat}(N, \mathbb{C})$ valued variables $S^{i}, S_{a b}^{i}=\xi_{a}^{i} \rho_{b}^{i}$ (see e.g. the papers [3, 1]). Such equations can be viewed as isotropic analogues of the equations (4.27)-(4.29). In our approach we deal with $\operatorname{Mat}(N M, \mathbb{C})$ valued variable $\mathcal{S}$, and $2 N M$-dimensional parametrization is given not by the pair of rectangular matrices of size $N \times M$, but rather arises as a particular case (of rank 1) for the matrix of size $N M \times N M$, as in (15.2). 


\section{$6 \quad$ Elliptic functions}

The main tool for construction of the Lax pairs with spectral parameter on elliptic curve $\Sigma_{\tau}$ with moduli $\tau(\operatorname{Im}(\tau)>0)$ is the Kronecker function

$$
\phi(z, q)=\frac{\vartheta^{\prime}(0) \vartheta(z+q)}{\vartheta(z) \vartheta(q)}
$$

defined via the Riemann theta-function

$$
\vartheta(z)=\sum_{k \in \mathbb{Z}} \exp \left(\pi \imath \tau\left(k+\frac{1}{2}\right)^{2}+2 \pi \imath\left(z+\frac{1}{2}\right)\left(k+\frac{1}{2}\right)\right),
$$

which has a simple zero at $z=0$ due to its oddness. Also we use the first and the second Eisenstein functions

$$
E_{1}(z)=\frac{\vartheta^{\prime}(z)}{\vartheta(z)}, \quad E_{2}(z)=-\partial_{z} E_{1}(z)=\wp(z)-\frac{1}{3} \frac{\vartheta^{\prime \prime \prime}(0)}{\vartheta^{\prime}(0)},
$$

where $\wp(z)$ - is the Weierstrass $\wp$-function. The function $E_{2}(z)$ is double-periodic on the lattice $\mathbb{Z}+\tau \mathbb{Z}$ and has the second order pole at $z=0$. The first Eisenstein function and the Kronecker function have simple pole at zero with the residue equal to one. They transform on the lattice as follows:

$$
\begin{array}{cc}
E_{1}(z+1)=E_{1}(z), \quad E_{1}(z+\tau)=E_{1}(z)-2 \pi \imath, \\
\phi(z+1, q)=\phi(z, q), \quad \phi(z+\tau, q)=e^{-2 \pi \imath q} \phi(z, q) .
\end{array}
$$

The main relation for the function (A.1) is the Fay identity of genus one:

$$
\phi\left(z_{1}, q_{1}\right) \phi\left(z_{2}, q_{2}\right)=\phi\left(z_{1}-z_{2}, q_{1}\right) \phi\left(z_{2}, q_{1}+q_{2}\right)+\phi\left(z_{2}-z_{1}, q_{2}\right) \phi\left(z_{1}, q_{1}+q_{2}\right) .
$$

We use its degeneration for derivation of the Lax equation:

$$
\phi\left(z, q_{1}\right) \phi\left(z, q_{2}\right)=\phi\left(z, q_{1}+q_{2}\right)\left(E_{1}(z)+E_{1}\left(q_{1}\right)+E_{1}\left(q_{2}\right)-E_{1}\left(q_{1}+q_{2}+z\right)\right) .
$$

The following notation is used for the derivative of the Kronecker function with respect to the second argument:

$$
f(z, q)=\partial_{q} \phi(z, q) \stackrel{A .3}{=} \phi(z, q)\left(E_{1}(z+q)-E_{1}(q)\right) .
$$

For description of the models of elliptic tops we also define the set of function numerated by the index $\alpha=\left(\alpha_{1}, \alpha_{2}\right) \in \mathbb{Z}_{N} \times \mathbb{Z}_{N}$ in accordance with numeration of elements of the matrix basis (3.1):

$$
\varphi_{\alpha}\left(z, \omega_{\alpha}+\eta\right)=\exp \left(2 \pi \imath \frac{\alpha_{2}}{N} z\right) \phi\left(z, \omega_{\alpha}+\eta\right), \quad \omega_{\alpha}=\frac{\alpha_{1}+\alpha_{2} \tau}{N}
$$

Similarly,

$$
f_{\alpha}\left(z, \omega_{\alpha}+\eta\right)=\exp \left(2 \pi \imath \frac{\alpha_{2}}{N} z\right) f\left(z, \omega_{\alpha}+\eta\right) .
$$

Due to (A.7) the set of functions (A.9) satisfies the following relations:

$$
\begin{gathered}
\varphi_{\alpha}\left(z, \omega_{\alpha}+q_{1}\right) \varphi_{\beta}\left(z, \omega_{\beta}+q_{2}\right)= \\
=\varphi_{\alpha+\beta}\left(z, \omega_{\alpha+\beta}+q_{1}+q_{2}\right)\left(E_{1}(z)+E_{1}\left(\omega_{\alpha}+q_{1}\right)+E_{1}\left(\omega_{\beta}+q_{2}\right)-E_{1}\left(z+\omega_{\alpha+\beta}+q_{1}+q_{2}\right)\right) .
\end{gathered}
$$




\section{Acknowledgment}

This work is supported by the Russian Science Foundation under grant 19-11-00062.

\section{References}

[1] G.E. Arutyunov, S.A. Frolov, J. Phys. A31 (1998) 4203-4216; arXiv:hep-th/9703119.

[2] G. Arutyunov, E. Olivucci, arXiv:1906.02619 [hep-th]. G. Arutyunov, R. Klabbers, E. Olivucci, JHEP 05 (2019) 069; arXiv:1902.06755,

[3] E. Billey, J. Avan, O. Babelon, Physics Letters A, 186 (1994) 114-118; hep-th/9312042.

E. Billey, J. Avan, O. Babelon, Physics Letters A, 188 (1994) 263-271; hep-th/9401117.

I. Krichever, O. Babelon, E. Billey, M. Talon, Amer. Math. Soc. Transl. (2) Vol. 170 (1995) 83-120.

[4] O. Chalykh, M. Fairon, arXiv:1811.08727 [math-ph].

M. Fairon, arXiv:1811.08717 [math-ph].

[5] L. Fehér, arXiv:1901.03558 [math-ph].

L. Fehér, arXiv:1809.01529 [math-ph].

[6] J. Gibbons, T. Hermsen, Physica D: Nonlinear Phenomena, 11 (1984) 337-348;

S. Wojciechowski, Physics Letters A, 111 (1985) 101-103.

[7] A. Grekov, I. Sechin, A. Zotov, JHEP 10 (2019) 081; arXiv:1905.07820 [math-ph].

[8] I. Krichever, A. Zabrodin, Russian Math. Surveys, 50:6 (1995) 1101-1150; arXiv:hep-th/9505039.

[9] A. Levin, M. Olshanetsky, A. Zotov, Commun. Math. Phys. 236 (2003) 93-133; arXiv:nlin/0110045

[10] A. Levin, M. Olshanetsky, A. Zotov, JHEP 07 (2014) 012; arXiv:1405.7523 [hep-th]. G. Aminov, S. Arthamonov, A. Smirnov, A. Zotov, J. Phys. A: Math. Theor. 47 (2014) 305207; arXiv:1402.3189 [hep-th].

T. Krasnov, A. Zotov, Annales Henri Poincare, 20:8 (2019) 2671-2697; arXiv:1812.04209 [math-ph].

[11] A. Levin, M. Olshanetsky, A. Zotov, J. Phys. A: Math. Theor. 49:39 (2016) 395202;

arXiv:1603.06101 [math-ph].

[12] A. Levin, M. Olshanetsky, A. Smirnov, A. Zotov, J. Phys. A: Math. Theor. $46: 3$ (2013) 035201;

arXiv:1208.5750 [math-ph]

[13] A.P. Polychronakos, Phys. Rev. Lett. 89 (2002) 126403; hep-th/0112141.

A.P. Polychronakos, J. Phys. A: Math. Gen. 39 (2006) 12793; hep-th/0607033

[14] V. Prokofev, A. Zabrodin, arXiv:1907.06621 [math-ph].

[15] N. Reshetikhin, Journal of Mathematical Sciences, 213:5 (2016) 769-785; arXiv:1509.00730 [math-ph].

[16] S.N.M. Ruijsenaars, Commun. Math. Phys., 110:2 (1987) 191-213.

[17] E.K. Sklyanin, Funct. Anal. Appl. 16:4 (1982) 263-270.

[18] A. Zotov, Modern Phys. Lett. A, 32:32 (2017), 1750169 , 22 pp., arXiv: 1706.05601

[19] A.V. Zotov, A.M. Levin, Theoret. and Math. Phys. 146:1 (2006) 45-52.

A.V. Zotov, A.V. Smirnov, Theoret. and Math. Phys., 177:1 (2013), 1281-1338.

A. Grekov, A. Zotov, J. Phys. A: Math. Theor. 51 (2018) 315202; arXiv:1801.00245 [math-ph]. 